\title{
Right cranial lung lobe torsion after a diaphragmatic rupture repair in a Jack Russell terrier
}

\author{
Terzo, E. ${ }^{1}$, Pink, J. ${ }^{2}$, Puggioni, A. ${ }^{1}$, Shiel, R. ${ }^{1}$, Andreoni, V. ${ }^{1}$ and McAllister, H. ${ }^{1}$ \\ ${ }^{1}$ University Veterinary Hospital, University College Dublin, Belfield, Dublin 4, Ireland \\ 2 Willows Referral Service, 78 Tanworth Lane, Solihull, United Kingdom
}

\begin{abstract}
A seven-year-old male Jack Russell terrier was presented with a history of coughing, generalised weakness and lethargy 10 days after an abdominal coeliotomy to repair a large diaphragmatic rupture. Thoracic radiographs demonstrated a soft tissue mass in the midcaudal right thoracic cavity. Ultrasonographic studies, bronchoscopy and subsequent exploratory thoracotomy confirmed a diagnosis of a right cranial lung lobe torsion (LLT), with an anomalous caudodorsal displacement of the affected lobe. LLT should be considered as a differential diagnosis for respiratory tract disease following diaphragmatic rupture repair.
\end{abstract}

Key words: diaphragmatic rupture, dog, lung lobe torsion

Irish Veterinary Journal

\section{Corresponding author:}

\section{Eloisa Terzo}

Diagnostic Imaging Unit

University Veterinary Hospital

University College Dublin

Belfield, Dublin 4, Ireland

$\mathrm{Ph}+35317166099$

Fax +3531716 6061

Email: eloisa.terzo@ucd.ie

\section{Introduction}

A seven-year-old male Jack Russell terrier was presented with a history of coughing, generalised weakness and lethargy 10 days after an abdominal coeliotomy to repair a large diaphragmatic rupture. Thoracic radiographs demonstrated a soft tissue mass in the mid-caudal right thoracic cavity. Ultrasonographic studies, bronchoscopy and subsequent exploratory thoracotomy confirmed a diagnosis of a right cranial lung lobe torsion (LLT), with an anomalous caudodorsal displacement of the affected lobe. LLT should be considered as a differential diagnosis for respiratory tract disease following diaphragmatic rupture repair.

Lung lobe torsion (LLT) is a rare and life-threatening condition in dogs, cats and humans, defined by a rotation of a lung lobe around its long axis, followed by a displacement and twisting of the lobar bronchus and vessels at the hilus or bronchovascular pedicle (Suter and Lord, 1984). Most affected dogs are reported to be large breeds with a deep narrow thoracic conformation such as Afghan hounds (Neath et al., 2000). LLT presents less frequently in small and toy breed dogs, with a relatively higher predisposition in pugs (White and Corzo-Menendez, 2000; Rooney et al., 2001; Murphy and Brisson, 2006). It is more common in males, and all ages may be affected (D’Anjou et al., 2005). The degree of rotation may vary from $90^{\circ}$ to approximately $580^{\circ}$ (Johnston et al., 1988).

The right middle lobe is the most commonly affected

lobe, followed by the left cranial lobe (Breton et al., 1986; D'Anjou et al., 2005; Murphy and Brisson, 2006). The right middle lung lobe is the most mobile owing to its narrow bronchovascular pedicle, its thin and long shape and its lack of attachment to the mediastinum, thoracic wall and adjacent right cranial and caudal lung lobes (Suter and Lord, 1984; Gallanger, 1993). Single right cranial LLT is particularly uncommon in small breed dogs (Suter and Lord, 1984; D’Anjou et al., 2005).

This case report documents an unusual torsion of the right cranial lung lobe in a seven-year old male Jack Russell terrier with a recent history of a diaphragmatic rupture repair.

\section{Case history}

Ten days following surgery for a right sided radial and short circumferential diaphragmatic rupture (Figures la and $\mathbf{1 b}$ ), a seven-year old Jack Russell terrier was admitted to the University Veterinary Hospital (UVH) of University College Dublin with an acute onset, one-day history of coughing, increased respiratory effort, weakness and lethargy. Physical examination revealed tachypnoea and a rectal temperature of $37.6^{\circ} \mathrm{C}$. Haematological and biochemical values were normal. Thoracic auscultation revealed decreased bronchovesicular sounds and muffled heart sounds over the right hemithorax. Radiographic examination of the thorax showed an increased soft tissue opacity in the right 

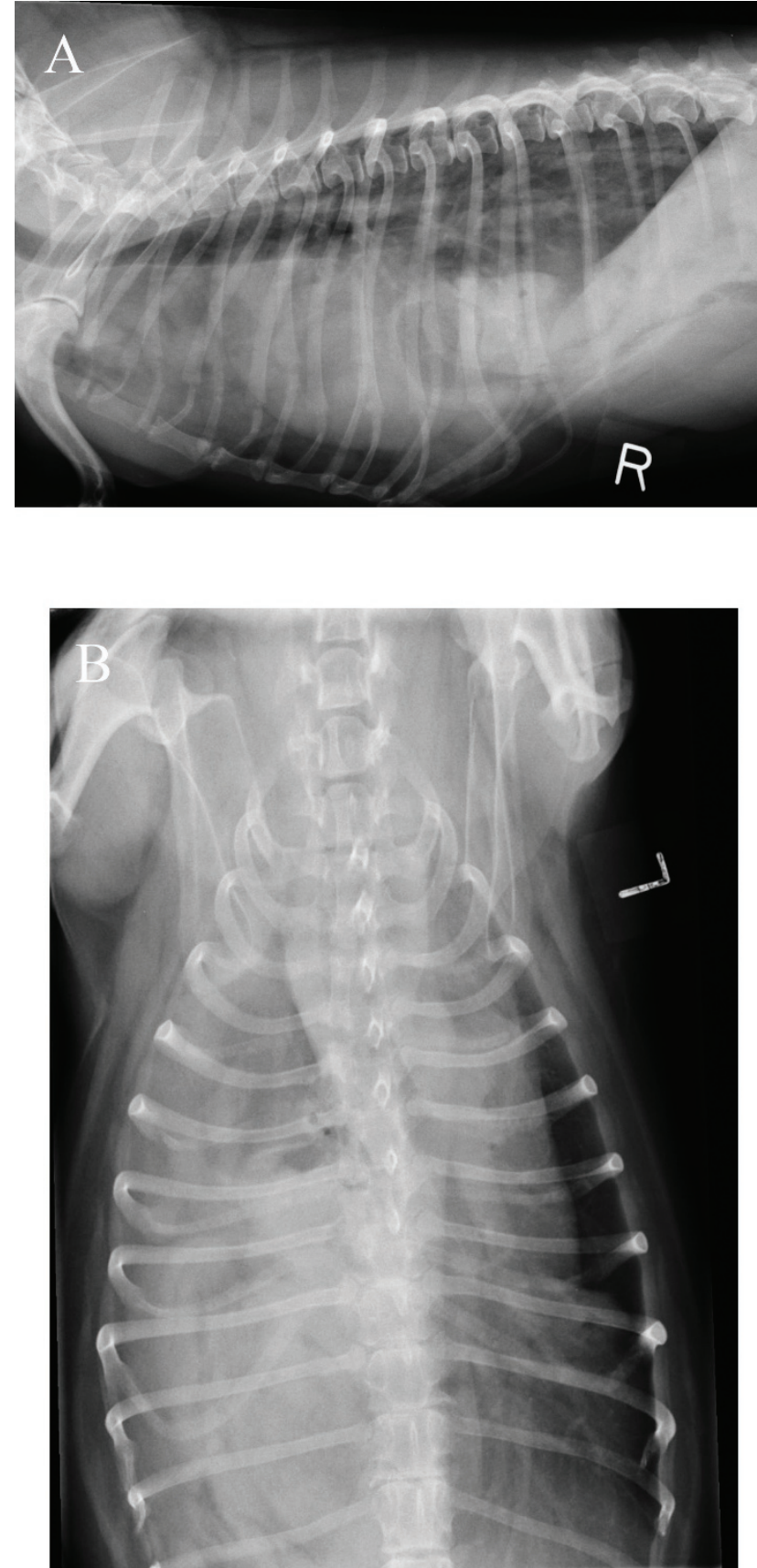

Figure 1: Lateral radiograph (a) showing herniation of abdominal organs into the thoracic cavity. Note the intestinal loops over the cardiac silhouette. Dorsoventral radiograph (b): Note the cranial displacement of the abdominal contents mainly in the right hemithorax.

hemithorax in the region of the right middle and caudal lung lobes (Figure 2a). In the dorsocaudal lung fields there was a mixed alveolar-interstitial pattern, with areas of consolidation and vesicular gas pattern, extending from the fifth to the ninth intercostal spaces along the right lateral thoracic wall. Medial to this area, there was a second soft tissue opacity with air bronchograms at the level of the right middle lobe, which was moderately displaced caudally from its normal position (Figure 2 b). In both right and left lateral recumbent inspiratory views, there was evidence of an interlobar fissure line in the right cranial thorax at the level of the second and third intercostal spaces, which was suspected to indicate a mild pleural effusion. Additionally,
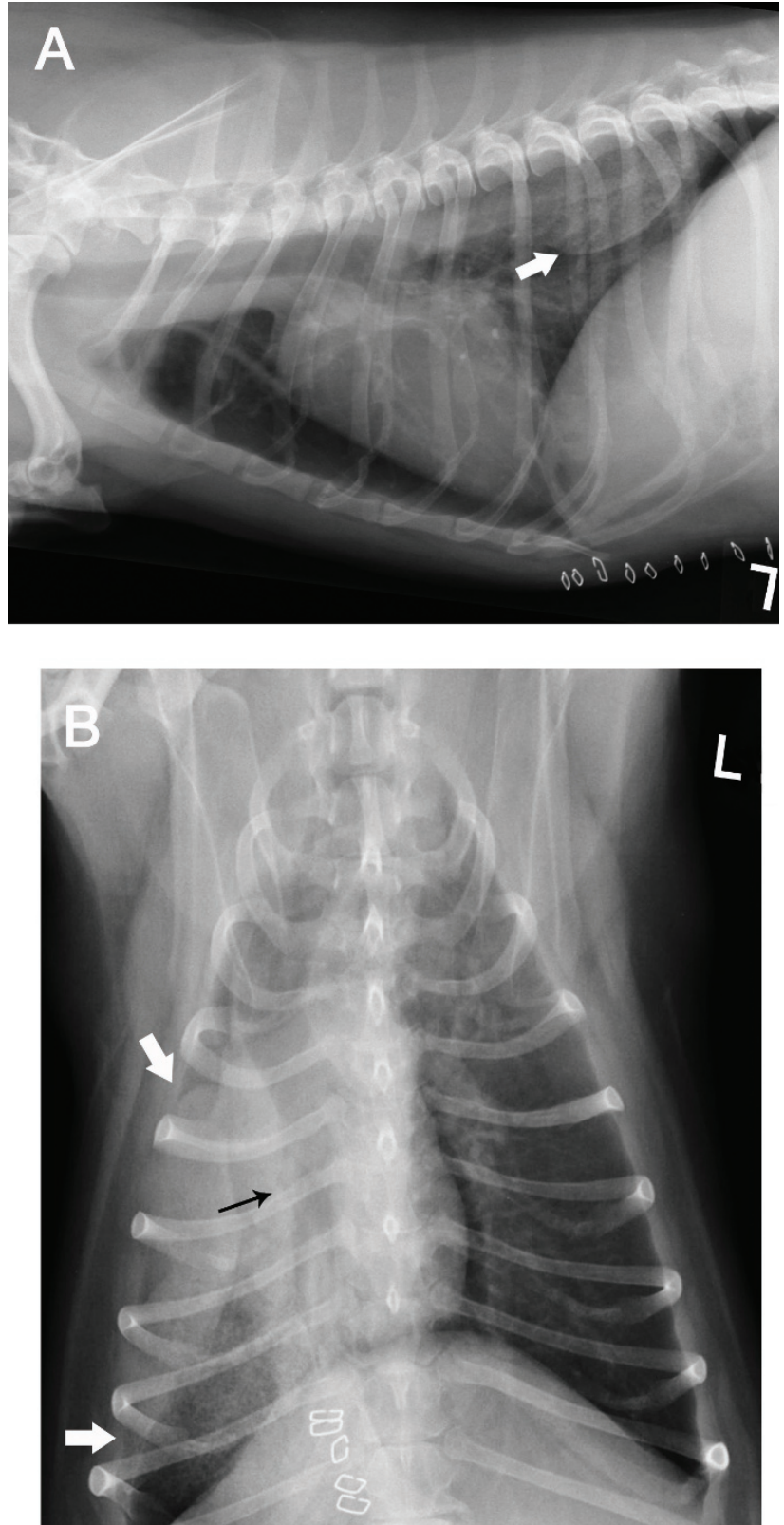

Figure 2: Lateral (a) and dorsoventral (b) thoracic radiographs of the same dog 10 days later. There is an increase in soft tissue opacity in the region of the right middle and dorso-caudal lung fields at the level of the fifth to ninth intercostal spaces (white arrows). The trachea is evident and displaced dorsally and kinked to the right at the level of the third intercostal space. There is also an axial rotation of the tracheal bifurcation. The right cranial lobar bronchus (black arrow in b) is displaced caudally to the level of the fifth intercostal space and is abruptly occluded.

the cardiac silhouette had a triangular shape, with a wellmarginated small gas radiolucency cranial and lateral to it, presumably caused by residual entrapped air within the mediastinum as a result of the previous coeliotomy. There was a pronounced right mediastinal shift, suggesting a decrease in the right middle lung lobe volume, a collapse of the right lung lobes or an obstruction of the right cranial and middle bronchi, being all possible conditions that might cause a mediastinal displacement toward the affected side (Kealy and McAllister, 2005). The terminal part of the trachea was widened, displaced dorsally and kinked towards the right at the level of the third intercostal space. There was also axial rotation of the carina (Figures $2 \mathbf{a}$ and $2 \mathbf{b}$ ). 


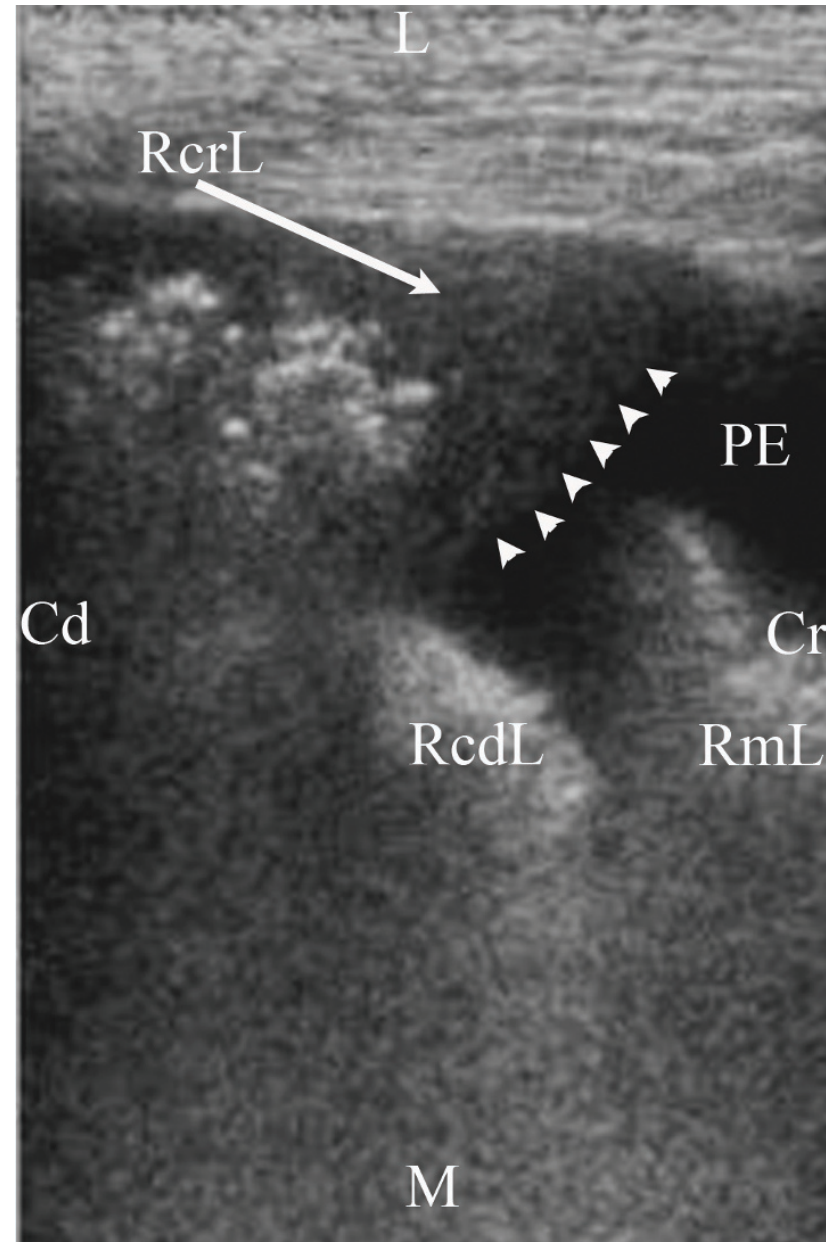

Figure 3: Ultrasonographic study (transverse plane) performed at the level of the fifth intercostal space of the right ventral thoracic wall. The right middle lobe $(\mathrm{RmL})$ has sharp margins, is small in size, atelectatic and retracted medially. The right caudal lobe $(\mathrm{RCdL})$ is partially aerated. Lateral to both of these lobes, along the thoracic wall, there is a mass in the right caudal hemithorax, with rounded margins, characterised by a liver-like echogenic tip and multiple gas bubbles reverberating in its central portion (arrow heads). This finding confirmed the corresponding vesicular gas pattern observed in the radiographs. This mass is the right cranial lung lobe ( $\mathrm{RCrL}$ ) (long arrow). An anechoic unilateral mild pleural effusion (PE) surrounds the lung lobes. (L: lateral; M: medial; Cd: caudal; Cr cranial.)

On the dorsoventral view, it was possible to follow the right and left mainstem bronchi and the lobar bronchi ramifications, except for a sudden blunting of the right cranial bronchus just distal to its origin. The right cranial bronchus was also caudally displaced (Figure $2 \mathbf{b}$ ). Radiographic differential diagnoses included: LLT; pneumonia; abscess; atelectasis; haemorrhage; diaphragmatic rupture; and, neoplasia.

Thoracic ultrasonography (Figure 3) was performed immediately after the radiographs, at the level of the fifth intercostal space of the right thoracic wall, and revealed that the right middle lobe had sharp margins, was small in size, atelectatic and retracted medially. The right caudal lobe was partially aerated. Lateral to both these lobes, there was a mass in the right caudal hemithorax, with rounded margins and characterised by a liver-like (hepatised) echogenic tip with multiple gas bubbles reverberating in its central portion. An anechoic unilateral mild pleural effusion surrounded the lung lobes.
Bronchoscopy (Figure 4) showed narrowing and occlusion of the right cranial bronchus with intraluminal serosanguinous fluid. The mucosa of the right cranial bronchus was folded with twisting of its folds in a clockwise direction, indicating a definitive diagnosis of right cranial lung lobe torsion.

The diagnosis was confirmed via a right fifth intercostal thoracotomy. The dog was premedicated with $3 \mathrm{mg} / \mathrm{kg}$ pethidine (Pethidine, BP Antigen Pharmaceuticals) and $0.2 \mathrm{mg} / \mathrm{kg}$ of midazolam (Hypnovel, Roche Pharmaceuticals) given intramuscularly. Anaesthesia was induced intravenously with $4 \mathrm{mg} / \mathrm{kg}$ of propofol (Rapinovet, Schering-Plough), followed by maintenance with isoflurane (Forane, Abbott) in 100\% oxygen and a fentanyl (Sublimaze, Janssen-Cilag Ltd) constant rate infusion at $0.4 \mu \mathrm{g} / \mathrm{kg} / \mathrm{hr}$. The lungs were mechanically ventilated. Fluid therapy (Compound Sodium Lactate, Pharmaceuticals) was infused at $10 \mathrm{ml} / \mathrm{kg} / \mathrm{hr}$. Perioperative amoxicillin (Augmentin, GlaxoSmithKline) 20mg/kg was administered intravenously.

The right cranial lung lobe was enlarged and congested. Its pedicle was torsed approximately $180^{\circ}$ clockwise, and the lobe was displaced caudally and dorsally into the right caudal hemithorax, compressing and pushing the consolidated right middle lobe medially. Cranial and middle lung lobectomies were performed. A 14 gauge thoracostomy tube was placed at the right ninth intercostal space (tunneled forward under the panniculus muscle from the eleventh intercostal space) and was removed 24 hours postoperatively. Intrapleural bupivacaine (Marcain, AstraZeneca UK Ltd) was placed after thoracotomy closure. The dog had an uncomplicated recovery and was discharged from the hospital two days postoperatively. The owner of the dog was contacted by telephone one month after surgery and confirmed that the patient was doing very well with no signs of respiratory tract problems.

\section{Discussion}

LLT may occur spontaneously, and affected dogs very often may not have evidence of underlying thoracic diseases (Spranklin et al., 2003; Hansen et al., 2006). A number of conditions have been suggested to predispose to LLT, including: thoracic trauma; pleural space disease; thoracic and abdominal surgery; mediastinal diseases; pulmonary parenchymal diseases; and, diaphragmatic hernia (D'Anjou et al., 2005).

The predominant clinical signs of LLT are: lethargy; progressive dyspnoea; coughing; anorexia; and, vomiting. Physical examination may reveal: pale mucous membranes; dull cardiopulmonary sounds on thoracic auscultation; pain on abdominal palpation; pyrexia; and, cyanosis (Murphy and Brisson, 2006).

Although many imaging studies can be useful in the diagnosis of LLT including radiography, ultrasonography, 

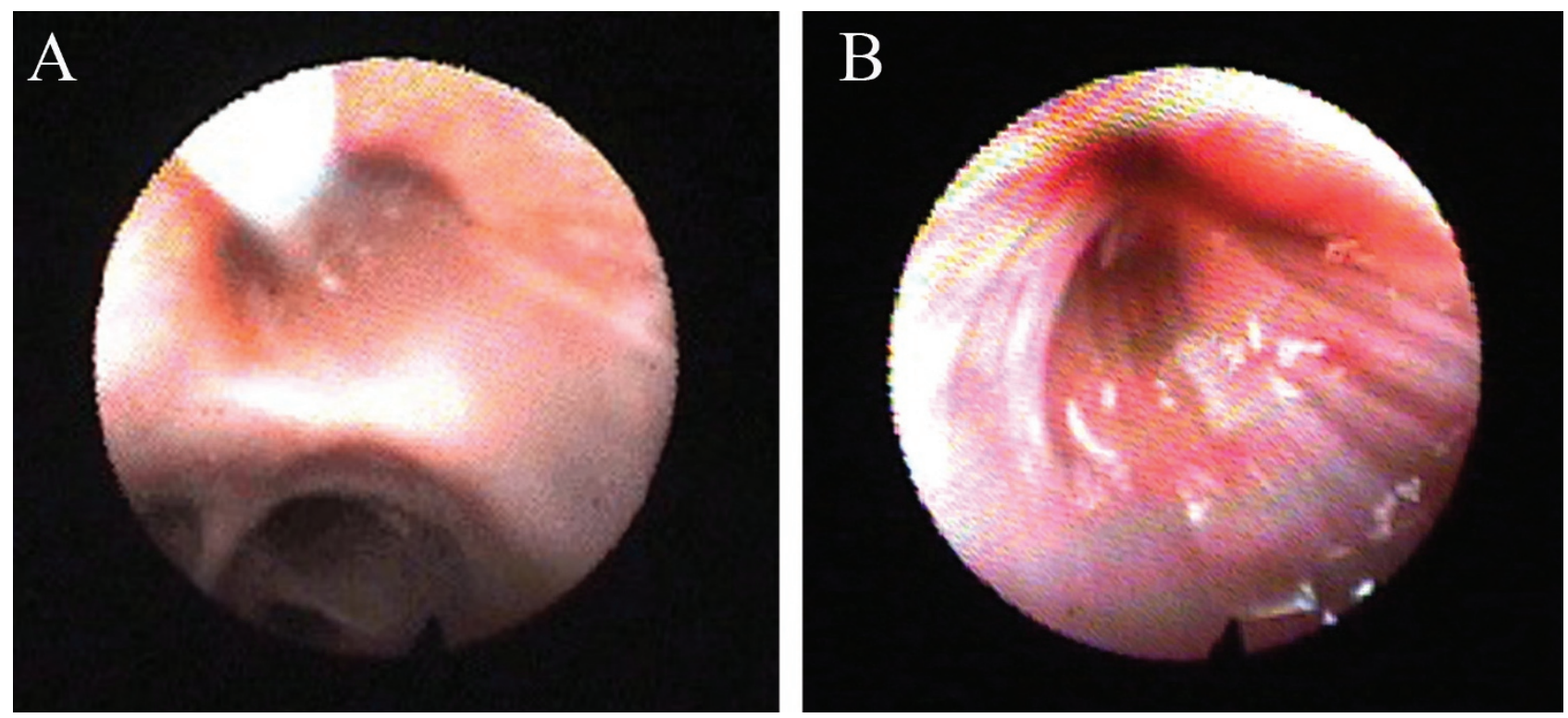

Figure 4: Bronchoscopy (a): the tip of the scope is located just beyond the carina, at the level of the right mainstem bronchus. The right cranial lobar bronchus is at 11 o' clock, the right middle bronchus is at 7 o'clock and both the caudal and accessory lobar bronchi are at 6 o'clock. The right cranial bronchus is occluded by a serosanguinous fluid and there i narrowing and occlusion of its lumen even after the introduction of a small amount of sterile saline. The other bronchi are patent.(b): the mucosa of the right cranial bronchus is folded, with twisting of the folds in a clockwise direction.

bronchoscopy, angiography, contrast bronchography, computed tomography (CT) and magnetic resonance imaging (MRI) (Spizarny et al., 1998; Reichle and Wisner, 2000; Kealy and McAllister, 2005), thoracoscopy and surgical exploration are currently performed for establishing a definitive diagnosis (Spranklin et al., 2003). In this paper, the authors describe the association of radiography, ultrasonography and bronchoscopy in establishing a presurgical diagnosis of LLT.

The patient developed a right cranial LLT 10 days after abdominal surgery for a large diaphragmatic rupture, with no known history of a recent acute traumatic episode. At the time of the surgery of the diaphragmatic rupture, visual inspection of the caudal thoracic cavity was unremarkable, with no adhesions between the organs, suggesting that the rupture was not chronic (Garson et al., 1980; Minihan et al., 2004).

Although diaphragmatic hernias may predispose to LLT (Rawlings et al., 1970), to the authors' knowledge there is no previous report of an associated torsion of the right cranial lung lobe following a diaphragmatic rupture repair. The pathophysiologic features of LLT are poorly understood. Deep interlobar fissures in dogs and cats facilitate the mobility of the lung lobes and predispose to the condition (Suter and Lord, 1984). Moreover, diseases that result in pleural effusion or pneumothorax may increase the space surrounding the affected lobe, predisposing to movement and possible torsion (Berkmen et al., 1989). It has also been suggested that the pressure exerted by herniated abdominal organs, or changes in intrathoracic pressure, could also play a role (Rawlings et al., 1970; Lord et al., 1973).

In the present case, it is postulated that the displacement of the abdominal organs into the right thoracic cavity caused severe compression and atelectasis of the right lung lobes. As a consequence, poor reinflation of these lobes after the resolution of the diaphragmatic rupture and the lack of pleural coupling associated with lung lobe instability, could have contributed to the right LLT. Unfortunately, postoperative radiographs after the diaphragmatic repair were not obtained, therefore this potential mechanism of action cannot be confirmed. Other predisposing factors may potentially have played an important role in this case. For example, an altered balance between production and absorption of pleural fluid, resulting in the production of a unilateral pleural effusion, coupled with concurrent ipsilateral mediastinal shift, may have contributed to the formation of the right cranial LLT.

Neutrophilia is the most common clinicopathological finding in LLT cases, although its significance is unknown (Murphy et al., 2006). However, in this case, haematological and biochemical values were normal. Caudal displacement of a torsed lobe and medial retraction of the adjacent lobe are other important findings of LLT, and usually the increased opacification in the area of the affected lobe helps to identify the involved lobe (D'Anjou et al., 2005). In this case, the lobe was abnormally displaced caudally and dorsally, leading to the suspicion in the first instance of an involvement of the right middle and caudal lobes.

As an exact radiographic location of LLT is difficult to assess, the most important radiographic feature that helped in the diagnosis of the right cranial LLT was the sudden blunted right cranial bronchus, which also appeared to be oriented more caudo-dorsally than expected. Moreover, ultrasonography helped in assessing the echostructure of the 'mass' and the amount of pleural effusion. Finally, bronchoscopy confirmed pre-surgically the diagnosis of torsion, as it showed the twisting of the bronchial mucosa of the right cranial bronchus. 


\section{Conclusion}

This is an unusual case since right cranial LLT, after a diaphragmatic rupture repair, has not been previously described in small breed dogs. It is important for clinicians to bear in mind that right cranial LLT may occur with an abnormal caudodorsal displacement, and thus should be considered a possible differential diagnosis in small breed dogs, especially after a diaphragmatic rupture repair or following any condition which disrupts and upsets the delicate equilibrium of the thoracic organs.

\section{Acknowledgements}

The authors would like to thank Dr Robert Furneaux for helpful assistance.

This paper follows an abstract presented at the annual meeting of the European Association of Veterinary Diagnostic Imaging (EAVDI), Hinxton (Cambridge, United Kingdom), October 13-14, 2006.

\section{References}

Berkmen, Y. M., Yankelevitz, D., Davis, S. D. and Zanzonico, P. (1989).

Torsion of the upper lobe in pneumothorax. Radiology 173: 447-449.

Breton, L., Di Fruscia, R. and Olivieri, M. (1986). Successive torsion of the right middle and left cranial lung lobes in a dog. Can Vet J 10: 386-388.

D'Anjou, M. A., Tidwell, A. S. and Hecht, S. (2005). Radiographic diagnosis of lung lobe torsion. Vet Radiol \& Ultrasound 46: 478-484.

Gallanger, L. A. (1993). Lung lobe torsion In: Disease Mechanisms in Small Animal Surgery, 2nd edn. pp 386-387. Philadelphia: Eds M. J. Bojrab, Lea \& Febiger.

Garson, H., Dodman, N. and Baker, G. (1980) Diaphragmatic hernia: analysis of fifty-six cases in dogs and cats. JSAP 21: 469-481.

Hansen, N. L., Hall, S. A., Lavelle, R., Christie, B. A. and Charles, J. A.

(2006). Segmental lung lobe torsion in a 7-week-old Pug. J Vet Emerg Crit

Care 16 (3): 215-218.

Johnston, G.R., Feeney, D.A., O’Brien, T.D., Klausner, J.S., Polzin, D.J., Lipowitz, A.J., Levine, S.H., Hamilton, H.B. and Haynes, J.S. (1984).

Recurring lung lobe torsion in three Afghan Hounds. J Am Vet Med Assoc 184: $842-845$.

Kealy, J. K. and McAllister, H. (2005). Lung lobe torsion In: Diagnostic Radiology \& Ultrasonography of the Dog and Cat. 4th edn. pp 195-198. Philadelphia: W. B. Saunders.

Lord, P.F., Greiner, T.P., Greene, R.W. and De Hoff, W.D. (1973). Lung lobe torsion in the dog. J Am Anim Hosp Assoc 9: 473-82.

Minihan, A. C., Berg, J. and Evans, K. L. (2004). Chronic diaphragmatic hernia in 34 dogs and 16 cats. J Am Anim Hosp Assoc 40: 51-63.

Murphy, K. A., and Brisson, B. A. (2006). Evaluation of lung lobe torsion in Pugs: 7 cases (1991-2004) J Am Vet Med Assoc 228: 86-90.

Neath, P. J., Brockman, D. J. and King, L. G. (2000). Lung lobe torsion in dogs: 22 cases (1981-1999). J Am Vet Med Assoc 217: 1041-1044.

Rawlings, C. A., Lebel, J. L. and Mitchum, G. (1970). Torsion of the left apical and cardiac pulmonary lobes in a dog. J Am Vet Med Assoc 156: 726733.

Reichle, J. K. and Wisner, E. R. (2000). Non-cardiac thoracic ultrasound in 75 feline and canine patients. Vet Radiol \& Ultrasound 41: 154-162.

Rooney, M. B., Lanz, O. and Monnet, E. (2001). Spontaneous lung lobe torsion in two pugs. J Am Anim Hosp Assoc 37: 128-130.

Spranklin, D. B., Gulikers, K. P. and Lanz, O. I. (2003) Recurrence of spontaneous lung lobe torsion in a Pug. J Am Anim Hosp Assoc 39: 446-451. Spizarny, D. L., Shetty, P. C. and Lewis J. W. Jr (1998). Lung torsion: preoperative diagnosis with angiography and computed tomography. Journal of Thoracic Imaging 13: 42-44.

Suter, P. F. and Lord, P. F. (1984). Lower airway and pulmonary parenchymal diseases. In: Thoracic Radiography: A Text Atlas of Thoracic Diseases of the Dog and Cat. Westwill STA edn. pp 635-640.

White, R. N. and Corzo-Menendez, N. (2000). Concurrent torsion of the right cranial and right middle lung lobes in a whippet. J Small Anim Pract 41: $562-565$. 